\title{
Charged colloidal particles in a charged wedge: do they go in or out?
}

\author{
H Löwen ${ }^{1}$, A Härtel ${ }^{1}$, A Barreira-Fontecha ${ }^{2}$, H J Schöpe ${ }^{2}$, \\ E Allahyarov ${ }^{1,3}$ and T Palberg ${ }^{2}$ \\ ${ }^{1}$ Institut für Theoretische Physik II: Weiche Materie, Heinrich-Heine-Universität Düsseldorf, \\ Universitätsstraße 1, D-40225 Düsseldorf, Germany \\ ${ }^{2}$ Institut für Physik, Universität Mainz, D-55099 Mainz, Germany \\ ${ }^{3}$ Institute for High Temperatures, Russian Academy of Sciences, Izhorskaya 13/19, \\ 127412 Moscow, Russia
}

Received 14 March 2008

Published 10 September 2008

Online at stacks.iop.org/JPhysCM/20/404221

\begin{abstract}
Using real-space microscopy experiments, theory and computer simulation, we study the behaviour of highly charged colloidal particles which are confined between two highly charged plates forming a wedge geometry. Under low salt conditions it is experimentally observed that colloidal particles accumulate in the cusp of a wedge to form dense fluid or crystalline ordered structures. This behaviour is found for various cell geometries, salt concentrations and gravitational strengths, and even stays stable when additional convection is present in the system. An effort is made to understand this effect qualitatively on the basis of linear screening theory. For a single macro-ion, linear screening theory predicts an attractive 'trapping' force close to the cusp of the range of the Debye-Hückel screening length. The attractive force diverges logarithmically with decreasing distance of the macro-ion from the wedge cusp, while at large distances the force is repulsive. The results of the linear screening theory are confirmed by computer simulations of the primitive electrolyte model with explicit co- and counterions.
\end{abstract}

\section{Introduction}

Structure [1], dynamics [2] and phase transitions [3] in geometric confinement can be very different from their bulk counterparts. In particular, colloidal suspensions can easily be confined e.g. between two parallel glass plates, and therefore serve as excellent model systems for confined situations $[4,5]$. By changing the plate distance one can continuously switch from a three-dimensional bulk system to a two-dimensional (monolayer) system. Confining channels are the essential ingredient in microfluidic devices and the transport of colloidal particles and biomolecules through such channels is highly relevant to steer the throughput of microfluidic devices [6-8].

The set-up of almost parallel charged glass plates has been studied for decades to confine charged colloidal suspensions and watch their structure and dynamics by videomicroscopy [9, 4, 10-12]. Likewise, corresponding theoretical studies on the structure and phase behaviour (see e.g. [13-15]) have been performed. Introducing a slight tilt angle between the plate orientation leads to a wedge confining geometry where the local plate-to-plate separation depends on the distance to the wedge cusp. The wedge geometry has been extensively used to perform experiments at different plate separations by just moving out of the wedge and changing the local plate separation slowly [9, 4]. For very small wedge angles, the plate separation changes extremely slowly with distance and a mapping of the structure and phase behaviour to that of parallel plates with the local distance is possible [16]. As a side remark, a wedge geometry is also considered in [17] but there the wedge was filled with DNA which is flexible and accumulation might be dominated by different mechanisms there.

In the present paper, we investigate the motion of charged colloidal particles at low salt concentration in a confining charged wedge. The crucial question we address is: is a colloidal particle attracted towards the inner of the wedge or is it expelled from the wedge? Our basic motivation to do so is twofold: first, this question is of central importance to understanding the transport of macromolecular particles in a charged channel of arbitrary geometry. One may use an understanding of the mechanisms to fabricate microfluidic devices to guide colloidal particles and/or to separate mixtures of the latter. The second motivation has to do with the still 
controversial discussion about like-charge attraction [18]. If charged particles accumulate in the wedge cusp it is tempting to investigate whether the underlying mechanism has to do with like-charge attraction.

As a result, we find in our experiments that particles do accumulate and get trapped in the inner cusp of a wedge. In addition a slow drift and the formation of a depletion zone adjacent to the accumulation zone are observed. Hence a local attractive force has to be present towards the inner wedge cusp. This effect is stable when studied for different geometries, salt concentrations, gravitational strengths, and even is persistent when additional convection is opposing the drift, leading to a state where colloidal particles stay in the wedge cusp, while regions further out are cleared of colloids. Some properties of the experimental findings are qualitatively explained within linear screening theory and computer simulations with explicit micro-ions. Our theory predicts a local attractive trapping force which draws particles further into the wedge and which diverges logarithmically with the distance of the particle to the wedge cusp. The range of the attraction is of the order of the Debye screening length. The linear screening theory is quantitatively confirmed by primitive model computer simulations. Consequently the effect has nothing to do with like-charge attraction, in fact, it is fully consistent with likecharge repulsion $[19,20]$.

One important implication of our findings is that edges and cusps are crucial for colloidal transport through microchannels since they lead to particles being trapped in the corners which may block efficient particle propagation. Furthermore, on micro-rough charged surfaces particles may get stuck in deep holes of the substrate due to the attraction mechanism explained in this paper.

Our paper is organized as follows. In section 2, the experimental set-up and the findings are described. Section 3 contains the linear screening theory and both numerical and analytical results obtained from this theory. Primitive model computer simulation data are provided in section 4 . We finally discuss and conclude with section 5 .

\section{Experiments}

\subsection{Samples and cells}

In two previous studies on layering transitions and on crystallization in confinement we have already noted the existence of a peculiar drift effect present in low ionic strength suspensions of charged spheres confined to a wedge geometry [21, 22]. In short, we observe a slow migration of particles towards the narrow part of a wedge where they form a region of enhanced particle density, which apparently is above the freezing density. Crystals are arranged in structures which sometimes are derived from close packed situations which are known from the bulk, but there are also interesting additional structures, such as buckled phases, rhombohedric phases, prism structures or close packed structures of unusual orientation. These structures have been interpreted as finite temperature equilibrium structures in restricted geometry and earned considerable theoretical and experimental interest. In a crystallization study of other authors performed under similar conditions and on the same structures as in [22] the migration effect, however, was not mentioned [23]. It also was not reported from the pioneering works of, e.g. Pieranski or Murray who used wedge geometries [24, 9]. Migration effects in bulk suspensions are well known from electrokinetics in external electric fields (electrophoresis) or gradients of salt concentration (diffusiophoresis) [25]. They are also known from gradients in solvent composition [26] or from gradients of temperature [27]. For confined suspensions, in particular, additional evaporation currents play a crucial role [28]. For large particles especially, sedimentation has to be considered, too. It was therefore interesting to have a systematic look at the observed effect and check for its reproducibility under various conditions as well as its equilibrium character. Additionally we seek to explore its possible cause in close collaboration with theory without evoking like-charge attraction types of arguments.

The accumulation effect has now been observed for different charged sphere species with diameters ranging between $500 \mathrm{~nm}$ and $1.7 \mu \mathrm{m}$. Particles were of different surface chemistry including carboxylated particles, particles stabilized by sulfate or sulfonate groups as well as silica particles carrying silanol groups on the surface. Polydispersities were of the order of $2-8 \%$. All these species were suspended in low salt or deionized water. Stock suspensions typically had been stored over mixed bed ion exchange resin for at least a few months before diluting these with distilled water to attain the desired starting particle densities. Particle densities were chosen to obtain volume fractions of the order of a few per cent or lower. The samples were left in contact with air to equilibrate the carbon dioxide content before filling them into the cells. For a few samples a further elevated salt concentration was adjusted by adding small amounts of 1:1 electrolyte (typically $\mathrm{NaCl}$ ). The accumulation effect was observed for all these species, irrespective of size, polydispersity and charge state.

To check for most of the above-mentioned fields and gradients, different cells were designed. These are listed in table 1. The effect was first observed in wedge cells (W), which consist of two standard microscopy slides glued together with a $50 \mu \mathrm{m}$ spacer at one side. A schematic drawing of this cell type is given in figure 1(a). Length $(70 \mathrm{~mm})$, height $(0<h<50 \mu \mathrm{m})$ and width $(25 \mathrm{~mm})$ correspond to $x-$, $y$ - and $z$-direction. The resulting wedge angle is $1 \mathrm{mrad}$. Typically epoxy glue was employed for sealing (W1, W3, W4) but also a silicone base fat (Glisseal HV, Borer, Germany) was tested (W2). Cells were assembled in the dry state with two small holes at the narrow and wide end left open in the sealing. Then a drop of suspension was applied to the spacer side and within a few minutes the suspension was sucked into the cell by capillary forces. Gentle shaking removed most remaining air bubbles. When the suspension had filled the cell both holes were sealed, too (W1-W3). This defined the beginning of the experiments: $t=0$. For W4 only the spacer side hole was sealed, leaving a small opening for evaporation into a large constant humidity chamber. Evaporation currents were found to be best adjustable by changing the humidity. 
Table 1. Characteristic properties of cells used (for further details see the text). PMMA: polymethylmetacrylate. Dimensions given are the length $\ell$ and width $w$ for the rectangular W cells. For W cells also the spacer height is noted. For L1 we give the diameter of the cell as well as the distance between the curved and plane plate at the perimeter in units of $\mu \mathrm{m}$. The angle at contact denotes the wedge angle at a cell height of $1.1 \mu \mathrm{m}$. For $\mathrm{L} 2$ we give the outer diameter of the upper lens and the inner diameter of the container as well as the spacing between lens and container bottom at the perimeter of the lens.

\begin{tabular}{lllllll}
\hline & & Plate & & Dimensions \\
& Type & material & Sealing & $\begin{array}{l}\text { Angle at } \\
\text { contact } \\
(\mathrm{mrad})\end{array}$ & Air contact \\
\hline W1 & Rectangular wedge & Glass & Glue & $70000 \times 25000 \times 50$ & 1 & No \\
W2 & Rectangular wedge & Glass & Grease & $70000 \times 25000 \times 50$ & 50 & No \\
W3 & Rectangular wedge & Quartz & Glue & $70000 \times 25000 \times 50$ & 1 & No \\
W4 & Rectangular wedge & Glass & Glue & $70000 \times 25000 \times 50$ & 1 & Evaporation leak \\
W5 & Rectangular wedge & Gold plated & Glue & $70000 \times 25000 \times 50$ & 1 & No \\
L1 & Circular wedge & PMMA & Viton & 90000,200 & $<1$ & Small bubbles \\
L2 & Circular wedge & Glass & Air/grease & $40000,50000,5500$ & $>1$ & Large reservoir \\
\hline
\end{tabular}

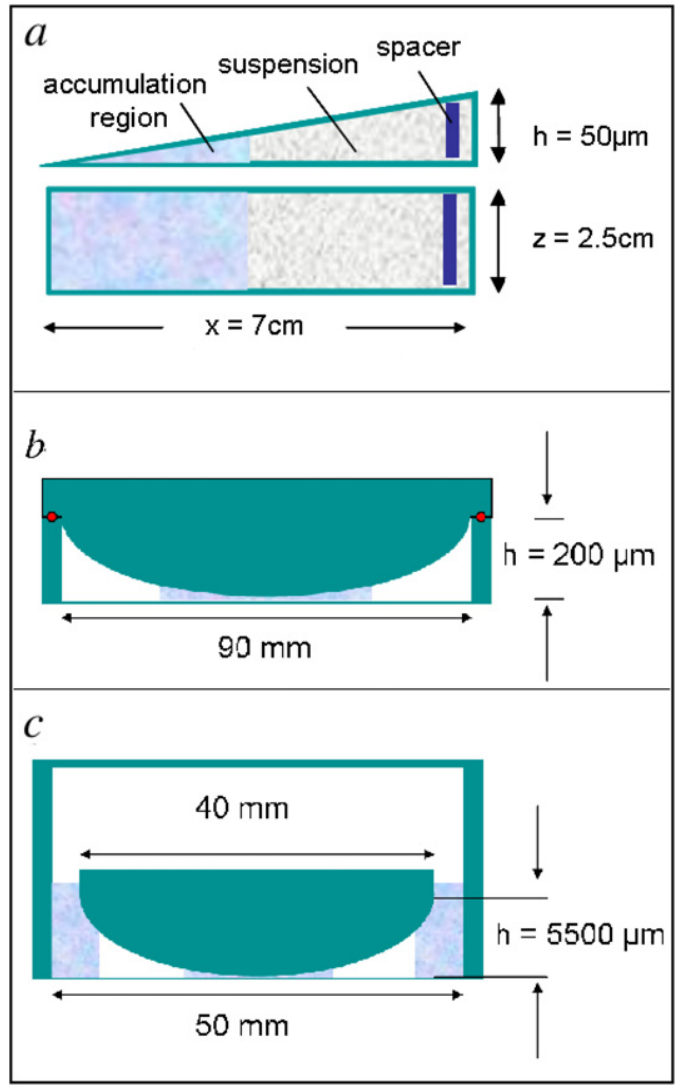

Figure 1. The three cell types investigated: (a) standard wedge cell $\mathrm{W}$ in side and top view; (b) L1-cell side view. In both cases the particles migrate to the narrow parts of the cells and form a densified region of crystalline structure, as indicated by the darker shading. (c) L2-cell side view; here a particle depleted region is observed which is sharply bound to both the inner densified region and an outer region of fluid structure which is approximately at the initial density. For details see the text.

As described below in more detail, several further variations of experimental conditions were performed concerning cell positioning, initial salt concentration, particle density, external fields and homogeneity of the initial state.

A second type of cell was constructed from lens-like upper plates and a flat bottom plate. In this cell type a spatially varying wedge angle is obtained. L2 was made simply by placing a $40 \mathrm{~mm}$ diameter optical plane-convex lens in a flat bottom beaker of $50 \mathrm{~mm}$ inner diameter. The cell was filled up to approximately half the side height of the lens and then the beaker was capped by a cover glass and sealed against the ambient air using fat. L2 contains a comparably large amount of initially low humidity air in contact with the large suspension surface. Thus, after sealing, evaporation and vapour pressure equilibration occurs, resulting in additional flow fields for the confined suspension. To keep the principal geometry, but minimize evaporation flow (and in addition check for the influence of salinity gradients) we designed a very small angle lens cell L1. This consisted of a $90 \mathrm{~mm}$ diameter lens-type upper plate put into contact with a plane bottom plate. The radius of curvature was chosen to be $50626 \mathrm{~mm}$ in order to obtain a small wedge angle over larger areas. L1 was filled using a standard peristaltic preparation set-up [29]. In L1 no contact with air is given except for a few remaining air bubbles. The volume of air is orders of magnitude smaller than in L2 and no evaporation currents were observed.

\subsection{The general effect}

Accumulation and migration effects were observed in $\mathrm{W}$ and $\mathrm{L}$ cells irrespective of experimental details. In all cases the initially homogeneous suspension started assembling at the narrow part of the wedge, forming densely packed (crystalline) regions which increased in lateral extension with time and showed the theoretically expected height dependent structures. In figure 2(a) we show the interface between the densified and the depleted region at a position corresponding to the height of three layers of crystalline structure. For this image the objective aperture was nearly closed, which results in loss of resolution for the coherently diffracting crystalline regions and the generation of a colour coding [30]. Two different stackings-ABA corresponding to hexagonally close packed structure and $\mathrm{ABC}$ corresponding to face centred cubic structure-are discernible by the different colouring. A high spatial resolution is retained for the regions of low density. In figure 2(b) we show a close-up of the interface at a position corresponding to the formation of a crystalline bilayer. Note that the crystalline regions are covered with a thin film of 

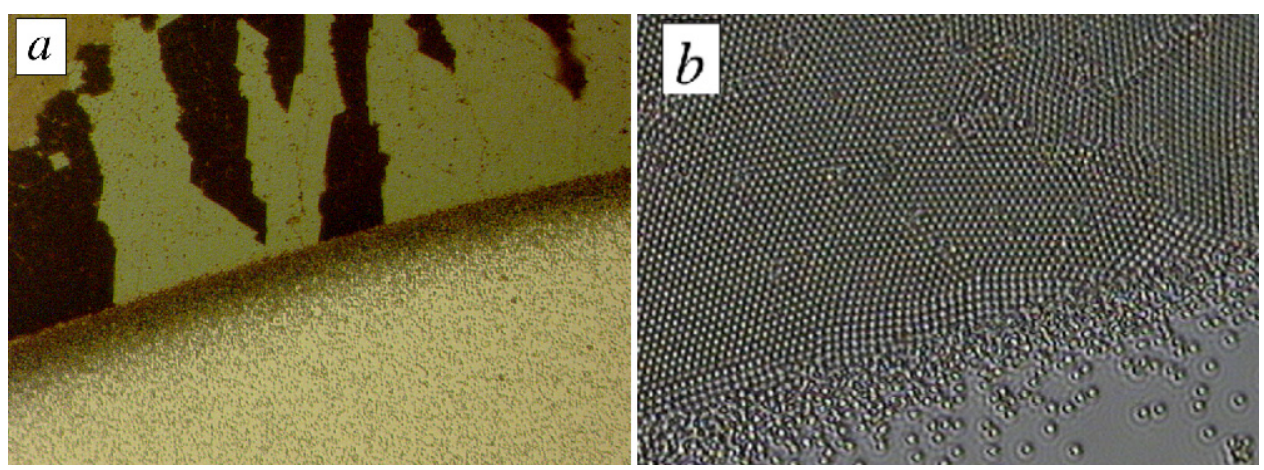

Figure 2. (a) Growth of a three layer close packed crystal in a W1 cell. The narrow part is at the top. Particles arrive from the bottom and first form a densified fluid layer. The increased attenuation of transmitted light shows the evolved density gradient. For sufficient density the fluid transforms to a crystalline structure with the interface progressing by $1-10 \mu \mathrm{m} \mathrm{h}^{-1}$. Differences in the colour of different crystal domains result from the two different stacking sequences possible: ABA (dark) and ABC (bright). In the second crystal from the left a triangular dislocation line is seen. (b) Close-up on a growing hexagonal bilayer in a W1 cell.

fluid order and comparable density. Several crystals grow in parallel and share a common interface with the fluid layer. The thickness of this region was 15-20 particle diameters in (a) and 5-10 particle diameters in (b). The fluid layer extension increases slightly with cell height and initial particle concentration.

Adjacent to this region we observed more or less pronounced depletion zones for the convection free $\mathrm{W}$ cells and the L1 cell. Their extension continuously increased with time. In some cases of low initial particle density these depleted regions filled the complete remaining cell. In most cases this stage was not reached within a few months. The remaining part stayed homogeneous in density, usually without any noticeable particle migration. Also in very dilute suspensions video-microscopy of individual particles far off the wedge did not show a preferred drift direction which would dominate Brownian motion. This general scenario was observed with some variation in all cases, hence the experimental answer to our question seems to be: they go in.

\subsection{Evolution of the crystalline region}

Cells of L1-type are well suited to perform quantitative studies of the assembly process. In figure 3 we show a sequence of high resolution micrographs taken close to the cell centre over several days. This cell was filled by peristaltic pumping leaving the suspension under an elevated pressure and increasing the cell height. When the pressure was released the walls quickly returned to the original position, thereby trapping a few particles at the position of closest approach to the cell centre under pressure. This feature was nicely retained and served as a position marker. Comparing the position of the ring to the later evolving new line of closest approach, one notes that the cell centre had shifted by some $80 \mu \mathrm{m}$. Particles were first pushed out quite far by the particle free fluid from the inner region. Their return due to the accumulation effect is seen by following the position of a step in the transparency of the sample. In figure 4 we show the temporal evolution of the radial position of this step with respect to the new cell centre, which clearly shows the effect of the mechanical relaxation of the cell walls.
We define a new position of closest approach by its long time asymptotic value.

The most striking observation in figure 3 is the nearly perfect concentric arrangement of crystal structures. Variations of radial extensions rarely exceeded $10 \mu \mathrm{m}$ s. On further observation, some of the crystalline regions do show deviations from perfect symmetry. In figure 3 this is, for example, visible for the bright square structure region at the image bottom, appearing after some $80 \mathrm{~h}$. The irregularities increase by number with increasing radial position and are most pronounced for structures which do not directly derive from close packed bulk structures (fcc 111 and 100). This is conceivable, as the height interval in which these structures are stable decreases with layer number. This is aided by the radial increase of the local wedge angle and thus ever smaller lateral extensions for different structures are observable. This makes the irregularities in radial extension a very sensitive indicator of local deviations from ideal geometry.

The overall very good concentric arrangement is also seen below for situations of varying supply currents, i.e. differing lateral extension of the fluid layer. In line with this observation, the crystal growth velocity is constant in time. This is shown in figure 4(b) for a time interval of $1 \mathrm{~h}$ during which no change of the assembled structure occurred. An interfacial velocity of about $10 \mu \mathrm{m} \mathrm{h}^{-1}$ is observed in this case. Typical velocities were found to vary between one and some $20 \mu \mathrm{m} \mathrm{h}^{-1}$ depending on initial particle concentration, cell type and particle species and layer number. It does not strongly depend on crystal structure. By contrast, the total width (crystal plus dense fluid) of the accumulation zone may vary quite strongly depending on the supply current (see also below).

Bragg scattering shows that the density across a crystalline region does not vary by more than $10 \%$ with radial position. It does, however, increase with time. This is corroborated by local observations of particle motions about lattice places. This motion becomes more and more restricted with time. In turn the positions of the boundaries between adjacent crystal structures may shift inward. In addition the sample shows an increase of colour contrast with time, indicating an increase 


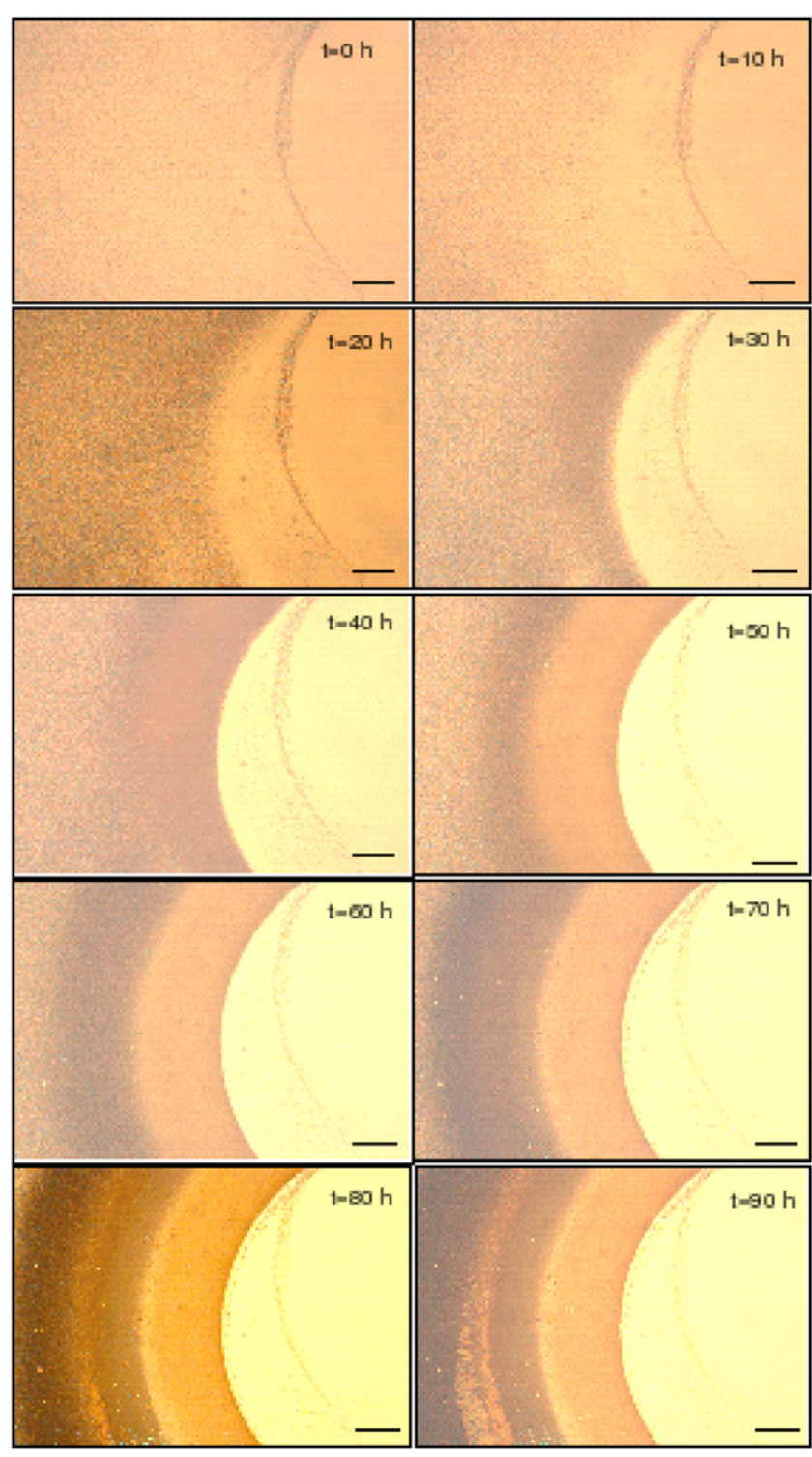

Figure 3. Sequence of images taken in an L1 cell for the different times indicated. The scale bar is $40 \mu \mathrm{m}$. The circular feature to the right of the actual accumulation zone is a ring of particles, which was trapped between the cell walls during filling under peristaltic pumping. Note that after release of pressure the distance of closest approach of particles to the centre and the centre position itself have shifted due to mechanical relaxation of the cell walls. The different colours again code different crystal structures and point defects are visible as bright spots. The regions of occurrence for different structures arrange concentrically and their lateral extension varies only slightly.

of crystal quality. Simultaneously we observe the appearance of point defects (voids) visible as bright spots accompanied by the disappearance of dislocation lines (not well seen here but in figure 2(a)). Similar behaviour has been found before in $\mathrm{W}$ cells under the influence of a laterally applied electric field [31]. We therefore believe that also here some annealing process takes place and it is interesting to question how this process couples to the overall drift.

After this general characterization of the accumulation and migration process and its effects on the structure formation
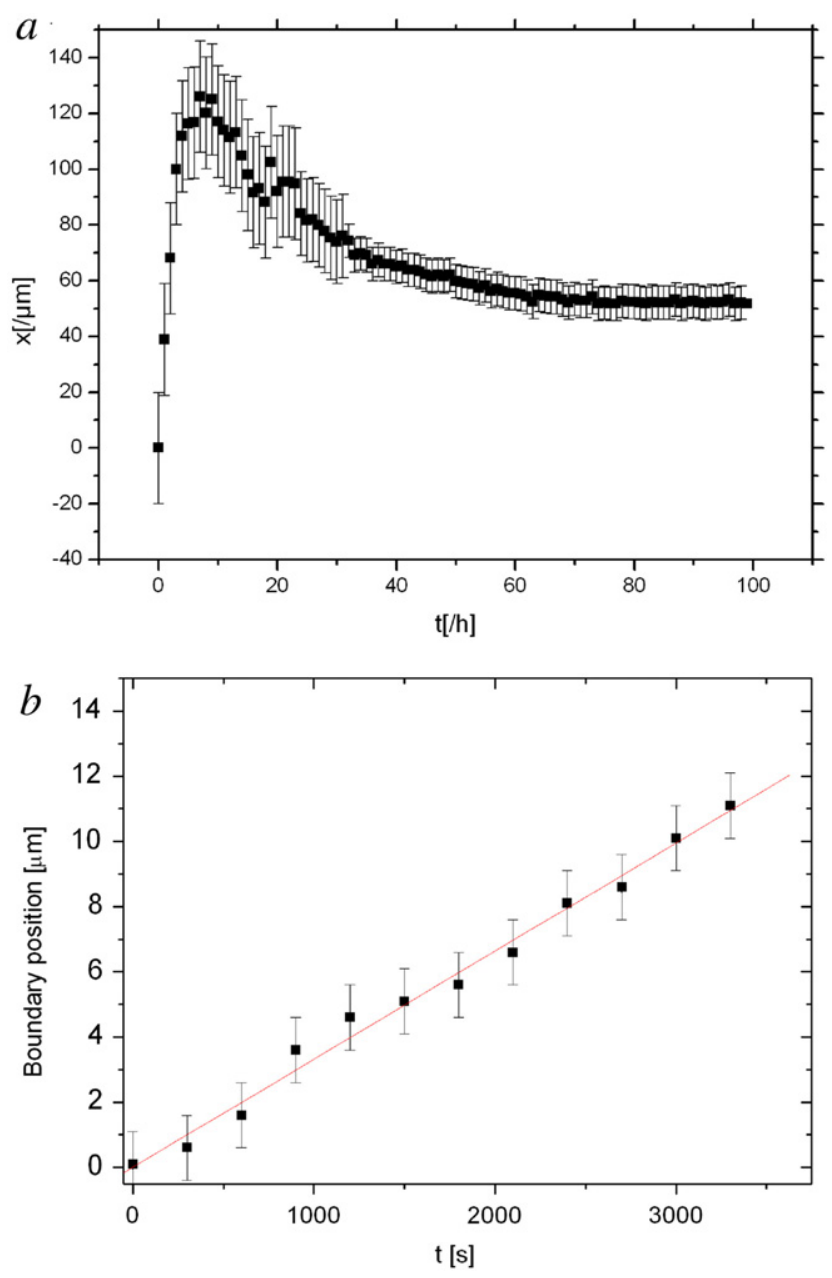

Figure 4. (a) Evolution of the radial distance of the inner boundary of the densified region to the cell centre with time after cell filling and end of pumping. The influence of mechanical relaxation of the cell walls is clearly seen. (b) Evolution of the crystal/fluid interface. The measurement started after $80 \mathrm{~h}$. A linear growth behaviour with a velocity of about $10 \mu \mathrm{m} \mathrm{h}^{-1}$ is observed.

in the accumulation zone, we shall now address some investigations undertaken to explore the underlying cause. The theoretical considerations below will address the case of a horizontally placed wedge and neglect gravity, salt gradients and convective flows. In addition, they consider the motion of an isolated sphere only. In the experiment we always work at finite density, so we cannot exclude the occurrence of interaction effects between particles. We can, however, exclude a number of other experimental artefacts, which have been observed to cause particle migration. In what follows we shall first address the influence of wedge wall charge, then of salt gradients and salt concentration, and finally of gravity and of additional convection counteracting or aiding the accumulation.

\subsection{Wall charge}

To test a possible influence of wall charges, different materials were employed in constructing the cells. W cells were built with glass and quartz walls, $\mathrm{C}$ cells in glass or plexiglass. 
These materials differ in their wall charge which develops in contact with water. In all cases we could observe the accumulation effect equally well. To obtain a larger charging effect, cell W5 was constructed in the usual way, but this time from glass plates sputtered with gold. The gold surfaces pointed to the suspension side and contacted outside the reach of the suspension with a conductive silver varnish. The cell walls could therefore be charged up to carry a surplus of positive or negative charge. Cells were filled the usual way and left for some time to obtain a crystalline structure. Then different voltages were applied. For positive voltages the particles strongly migrated into the slit and became deposited at various locations. The crystal structures collapsed to form dense amorphous lumps with occasional voids appearing. The evolving patterns were highly irreproducible but the samples were unstable in practically all cases.

For negative voltages the sample reaction depended on the start time. For short duration of the voltage free assembly period the crystals would simply melt via an increase of the lattice constant. For longer assembly times and in particular for multilayer crystals these would do the same at the crystalfluid interface. But deeper into the wedge crystals would float outward as a whole and, reaching a larger cell height, would disintegrate via a break-up into crystalline chunks. In some cases a switch of structure was observed during the outward drift, appearing like an adjustment to the increased height. While the richness of local processes was too large for a systematic investigation, the overall process of disintegration and outward drift was quite reproducible. An interesting point was the observation that a threshold voltage was needed to initiate the outward drift of whole crystals. Its magnitude varied, depending on the age of the sample. This possibly points at an increased trapping of crystals occurring in the course of time during the voltage free assembly.

\subsection{Salt gradients}

An often neglected, but sometimes essential source of particle migration may be the presence of salt gradients [32]. In deionized aqueous charged sphere suspensions the most important source of such gradients is the leakage of gaseous $\mathrm{CO}_{2}$ into the sample which there dissolves and partially dissociates to form hydrocarbonic acid. The equilibrium $\mathrm{pH}$ of deionized water exposed to air therefore is of the order of 5.5 and thus corresponds to an ion concentration of $5 \times$ $10^{-6}$ mol $\ell^{-1}$ per species. Thus considerable gradients can be created in a closed but gas leaky cell. In turn the effect of diffusiophoresis (which is a combination of interdiffusion and electrophoretic motion in local electric fields generated within the gradient [33]) leads to particle motion in these gradients. To be specific: if in a $\mathrm{W}$ cell the sealing were leaky, the largest gradients would occur in the $x$-direction and at the narrow side, where the solvent volumes are smallest. For the case of a highly mobile cation $\left(\mathrm{H}^{+}\right)$and a slow anion $\left(\mathrm{CO}_{3} \mathrm{H}^{-}\right)$a negatively charged particle will tend to migrate up the gradient, i.e. towards the narrow side of the wedge. By constructing a $\mathrm{W}$ cell $\mathrm{CO}_{2}$ may enter at all sides, but yield a larger concentration at the narrow side. Consequently any leakage of $\mathrm{CO}_{2}$ into the cell could cause a migration. By contrast, in an L1 cell gas can possibly only enter at the wide side. Thus there we would expect a diffusiophoretic motion towards the perimeter of this cell.

We prepared a sample of short contact time with air and filled a W1 cell with it. In addition the same sample was deionized thoroughly and directly filled into a L1 cell without intermediate contact with air. In both cases an accumulation was observed in combination with a migration towards the narrow cell part. Inward motion was with the gradient direction in $\mathrm{W}$ cells and against it in $\mathrm{C}$ cells. Thus the accumulation and migration are not influenced by gradients.

We further checked for the influence of a homogeneous salt concentration. At low concentrations the particle interactions are long ranged and the experimental suspension typically has evolved a fluid like short range order. Addition of salt to the suspension before filling the cell can diminish the particle interactions. We compared the migration behaviour for three different salt concentrations: $5 \times 10^{-6} \mathrm{~mol} \ell^{-1}\left(\mathrm{CO}_{2}\right.$ saturated), $1.5 \times 10^{-5} \mathrm{~mol} \ell^{-1}$ and $8.5 \times 10^{-5} \mathrm{~mol} \ell^{-1}$. In the three cases of still stable suspensions, the accumulation effect prevailed. It became somewhat less pronounced with increasing salt concentration, i.e. the border region became less sharp than in figure 2, the density in the accumulation region became smaller, and for the largest concentration only a fluid order was established. Thus the amplitude of the effect is influenced by the salt concentration and identifies it as of electrostatic origin. On the other hand, the accumulation became slower with increasing salt concentration, suggesting that the diminished repulsion between the particles renders the remainder of the cell more compressible.

\subsection{Gravity}

The influence of gravity was checked in several ways. First we used two W1 cells filled with suspensions of polystyrene particles (diameter $2 a=1200 \mathrm{~nm}$; mass density $\rho_{M}=$ $1.05 \mathrm{~g} \mathrm{~cm}^{-3}$ ). In one we used water as solvent, in the second a 1:1 mixture of water with deuterated water was employed. The accumulation effect was found to be present in both cases. It was possibly somewhat less pronounced in the buoyancy matched suspension, as there no crystals formed, while in the aqueous sample small crystallites were observable in the densified region at the narrow cell end. It is not clear whether this observation is solely due to the absence of gravity or whether slight differences in the dielectric constant of the different solvents also influenced the charge state of the two suspensions differently. A charge reduction would lead to an effect similar to an increased salinity and induce an accumulation region of fluid order.

Second, we performed tilt experiments. If a wedge cell was turned by $90^{\circ}$ about the $x$-axis from its usual horizontal placement, particles sediment to the lower side. Closer to the narrow part the sedimentation is less fast, possibly due to a combination of hydrodynamic slowing due to backflow with the migration effect. For long times practically all particles have sedimented. Reducing the tilt angle from $90^{\circ}$ sedimentation becomes less pronounced and finally ceases to cause a density gradient in the $y$-direction. 


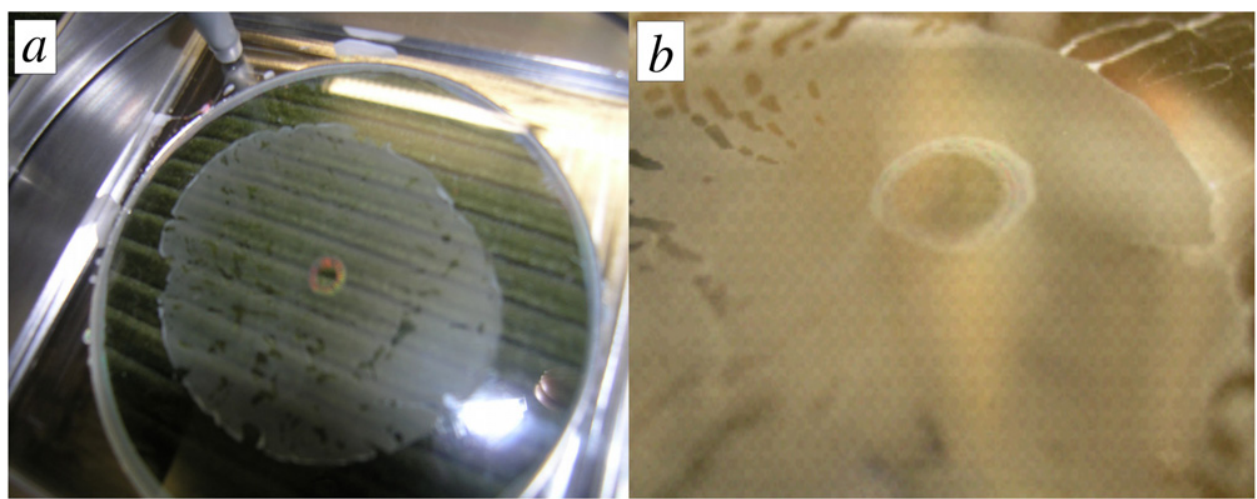

Figure 5. Accumulation effect in cell L1. (a) Large scale view showing parts of the assembly including one of the micrometre screws for height adjustment and the outer aluminium frame with circular Viton sealing. The stripy appearance of the inner optical part mirrors the laboratory ceiling and thus is an artefact. The central ring shows orange iridescence, indicating crystal formation. In this experiment the suspension fills about the central $75 \%$ of the cell. Deviations from sphericity of the outer suspension parts are due to the filling history. This cell was filled and kept horizontally. (b) For this experiment the cell was filled and then placed upside down (plane wall up) and slightly tilted. The lower cell side is to the left bottom of the image. Due to the tilt the suspension as a whole was shifted to the lower side, but the migration effect is present also in this case. One observes a particle free central contact zone of approximately $8 \mathrm{~mm}$ diameter where $h<2 a$, surrounded by an annular whitish region of densified suspension, which is distorted in the direction of tilt.

The influence of tilt in the $x$-direction is more difficult to study in $\mathrm{W}$ cells, as it runs parallel to the migration itself. If, however, the cell was turned such that gravity pointed towards the spacer, still quite a few particles were found in the cusp region even after two weeks, when the majority had settled at the bottom with the spacer. Particles were freely moving and not pinned to the wall, but the density of particles was too low to form an ordered phase. An observation pointing to the same direction was made using cell L2. Again an aqueous suspension was used. In figure 5(a) the standard situation is depicted: after filling, the cell was left in a horizontal position. With time a centro-symmetric crystalline ring forms and grows. In a second run the cell was filled and then placed upside down and in addition slightly tilted with a tilt angle of $5 \%$. Figures 5(a) and (b) show that the suspension as a whole had shifted towards the low side (bottom left in the image). The accumulation effect is present within the shifted suspension with a pronounced crystalline ring formation taking place. The lateral extension of the accumulation zone is larger at the upper side. This shows that the gravity-induced additional large scale flow of suspension had led to an additional accumulation, which is absent at the lee side. By comparison, the crystalline ring and the accumulation zone was symmetric in figure 5(a). This shows that the accumulation effect is present even in a small gravitational field, but can be enhanced or diminished by additional flow. Thus gravity does not play any direct role, rather it induces large scale flow, which alters the amplitude of the effect, but not the effect itself. The observation of particles in a gravity depleted cusp as well as the appearance of a depletion zone suggest that some kind of trapping by a local attractive well is the main cause of accumulation, rather than a migration towards the cusp caused by globally acting forces.

\subsection{Additional convection}

While gravity induced an additional uniaxial field where particles would move through a stationary solvent, also solvent flow can be thought of as a possible cause. This is obvious for the case of evaporation currents, which would transport particles towards the leak. A different type of solvent flow could be observed in the L 2 cells. The cell design in principle was the most straightforward realization of a wedge cell (cf figure 1(c)). However, here the suspension is in contact with a comparably large reservoir of air at initially ambient humidity. Figures 6(a)-(c) show a sequence of images taken on an L2 cell. Figure 6(a) shows the cell immediately after assembly and sealing, figure 6(b) after one day and figure 6(c) after two days. One observes in (a) the appearance of a diffuse zone of enhanced transmission, reflecting the varying optical path length. By contrast in (b) the borders of an inner clear region appear sharp. In (c) the borders appear again more diffuse and the transparent region has shrunk. In addition a whitish ring has formed in the central part. Closer inspection showed that this ring was of crystalline structure. At the upper lid condensate drops have formed.

The initial outward motion of particles and the formation of a sharply bordered transparent region is interesting. It is connected to another observation at the air suspension contact. As the air in the reservoir is initially at ambient humidity, evaporation of water into the reservoir occurs accompanied by the formation of a 'coffee-ring' at the air suspension interface. This ring-like drying pattern can be observed by eye if the cell is inspected from its side. It occurs at both the lens and the outer wall. Ring formation has been frequently observed in the drying of drops of colloidal suspensions in contact with air [34]. Also the formation of a central particle depleted zone has been frequently observed in several different systems drying in open dishes [35]; it was attributed there to the coupled action of sedimentation and evaporation currents. Also here the formation of the particle depleted region seems of the same origin. This is corroborated by the observation that the shape of the depleted zone may considerably deviate from sphericity, if the wetting conditions at the outside are not circular symmetric. In figures 7(a)-(d) we show a similar sequence as in figure 6 , 

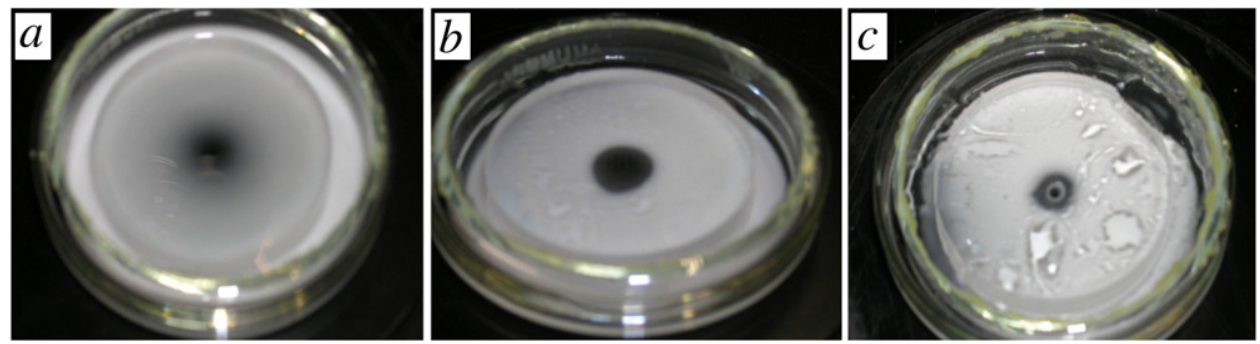

Figure 6. Sequence of images taken for a sample in an L2 cell. (a) Immediately after assembly one observes a smooth change of transparency due to the radially increasing optical path length; (b) after one day a centro-symmetric convection zone which appears to be depleted of particles has formed; (c) after two days the convection loses its sharply bordered appearance and a crystalline central ring has formed. The brighter spots in (b) and (c) are condensate drops forming at the lid.
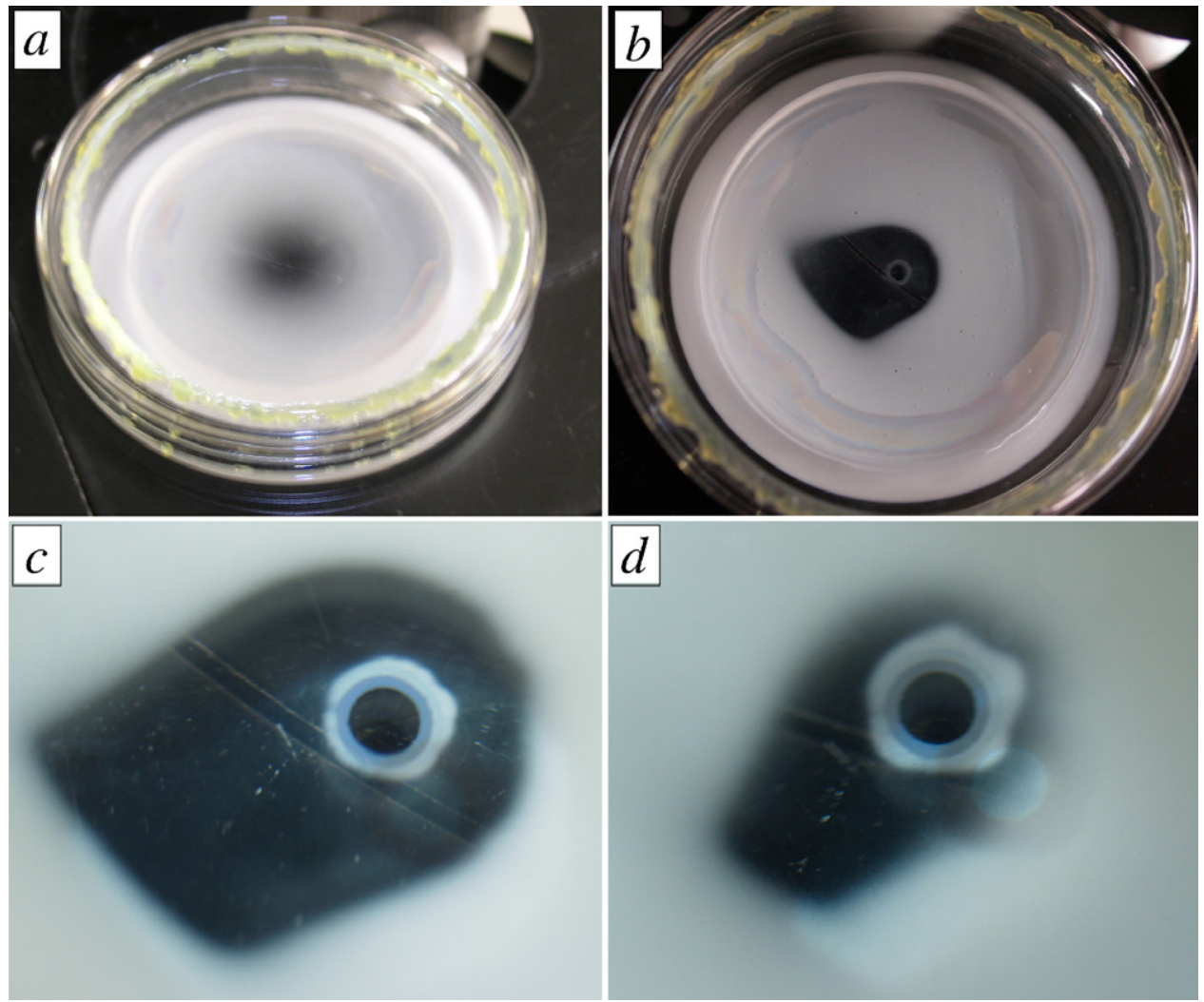

Figure 7. Sequence of images taken for a sample in an L2 cell which was filled to a lower height and slightly tilted. This resulted in a non-spherical meniscus. (a) As in figure 5(a) one initially observes a smooth change of transparency. (b) An asymmetric convection zone has evolved after $36 \mathrm{~h}$ and a ring has formed in the centre. (c) Close-up of the ring after three days. The borders of the particle depleted region have become diffuse. The asymmetric shape of the central densified region reflects the differences in supply currents. The two parallel lines are features of the sample stage. (d) After four days the particle free zone has shrunk to partially merge with the inner densified region. Note the perfect sphericity of the two concentric crystalline rings. The roundish feature to the lower right of the centre is a reflection at the cover lid.

but now taken in a slightly tilted cell which was filled to a lower height. Due to the tilt the suspension on one side wetted the outer perimeter of the lens, while at the opposite side it wetted the lens bottom only. Despite the same initial suspension conditions a lop-sided pattern evolved, again we observe a sharp border, which later shrunk asymmetrically. In turn also the subsequent particle accumulation in the centre deviated from sphericity, showing local differences in the supply currents. Interestingly the pattern of crystal structures does not react to this symmetry breaking. This is seen in figure 5(d), where one can discriminate two perfectly concentric iridescent rings of different colour. The crystal structure thus is determined only by the local height. Hence the density in the crystalline region is constant irrespective of the magnitude of the local supply current.

\subsection{Summary of experimental results}

So far the accumulation effect has been a useful tool to assemble particles in a slit and study their structure and 


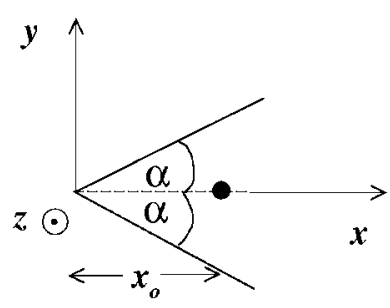

Figure 8. Sketch of the wedge geometry with total opening angle $2 \alpha$ and the distance $x_{0}$ of the macro-ion (full black circle) from the apex at the origin. The directions of the $x, y$ and $z$ axes are indicated as well.

dynamics in confinement. Here we undertook some initial systematic investigations into its origin. We found that the effect is very robust and occurs under various conditions of particle and salt concentration, for different wall materials. The speed of accumulation could be varied over a wide range by altering the supply currents, but even in the presence of counteracting sedimentation or convective currents the effect prevailed. Further, we could safely exclude a transport by solvent currents or within salt gradients towards the cusp, where particles simply would get stuck and thus accumulate due to a preceding migration. So from the experimental side the answer to the underlying question may now be given in more detail. Yes, they go in and accumulate, if they are sufficiently close to the cusp, while the ones further outward simply follow in order to balance the occurring depletion. This of course immediately raises the interesting question regarding the distance up to which the effect prevails, given a sufficient particle supply. Experiments along this line are under way.

\section{Linear screening theory}

In our theoretical approach, we simplify the geometry by assuming a perfect linear wedge form. The corresponding setup is shown in figure 8: two semi-infinite plates which are carrying a constant surface charge density $\sigma$ are forming a wedge with total opening angle $2 \alpha$. The apex of the wedge is placed at $x=0$ and $y=0$, the $z$-direction is the symmetry direction of the wedge perpendicular to the two-dimensional sketch of figure 8 . The broken line in figure 8 indicates the bisecting $x z$-plane of the wedge. We place a macro-ion of charge $Z$ inside this plane at a distance $x_{0}$ from the cusp line at $z=0$.

The goal is to calculate the effective potential $V\left(x_{0}\right)$ acting on the macro-ion as a function of $x_{0}$. We treat the problem using the linearized Debye-Hückel screening theory [36, 37]. Similar approaches based on Debye-Hückel and PoissonBoltzmann theory have been proposed in the literature, see e.g. [38, 39]. In [38] a more general geometry is treated and in [39] the line tension was calculated but in both references no additional macro-ion was considered. A neutral wedge was studied in $[40,41]$ filled with macro-ions but to the best of our knowledge there is no previous work for a macro-ion inside a charged wedge.

For simplicity we assume that there is a single inverse Debye-Hückel screening length $\kappa$ in the system which is fixed by the imposed salt concentration $c_{\mathrm{s}}$. We can include the counterions stemming from the walls (and from the macroion) in a later stage but their inclusion is not expected to qualitatively affect the linear screening theory but just to renormalize the inverse screening length.

Integrating over the full plate charge with the screened Coulomb potential involving a Debye-Hückel inverse screening length $\kappa$ results in

$$
\begin{aligned}
& V\left(x_{0}\right)=\frac{Z \sigma}{2 \pi \epsilon_{0} \epsilon} \\
& \quad \times \int_{0}^{\infty} \mathrm{d} y^{\prime} \int_{0}^{\infty} \mathrm{d} x^{\prime} \frac{\mathrm{e}^{-\kappa} \sqrt{x_{0}^{2} \sin ^{2} \alpha+\left(x_{0} \cos \alpha-x^{\prime}\right)^{2}+y^{\prime 2}}}{\sqrt{x_{0}^{2} \sin ^{2} \alpha+\left(x_{0} \cos \alpha-x^{\prime}\right)^{2}+y^{\prime 2}}} .
\end{aligned}
$$

Here $\epsilon$ is the dielectric constant of the solvent. An asymptotic evaluation of this integral yields the following two extreme limits.

(i) For large distances $x_{0} \rightarrow \infty$ the effective interaction in units of the thermal energy $k_{\mathrm{B}} T$ becomes

$$
\frac{V\left(x_{0}\right)}{k_{\mathrm{B}} T} \approx \frac{Z \sigma \lambda_{\mathrm{B}}}{\kappa \mathrm{e}^{2}} 4 \pi \mathrm{e}^{-\kappa x_{0} \sin \alpha}+\mathrm{O}\left(\mathrm{e}^{-\kappa x_{0}}\right),
$$

where $\lambda_{\mathrm{B}}=e^{2} / 4 \pi \epsilon_{0} \epsilon k_{\mathrm{B}} T$ denotes the Bjerrum length $\left(\lambda_{\mathrm{B}}=7.8 \AA\right.$ for water at room temperature) where $e$ is the elementary charge. Equation (2) implies that the force is repulsive for large distance, i.e. the macro-ion is excluded from the wedge. The typical exponential decay length is $1 / \kappa \sin \alpha$ for $0 \leqslant \alpha \leqslant \pi / 2$. The smaller the opening angle $\alpha$, the weaker the repulsion. Given the very small opening angle for the wedge in our experiments, the repulsion is very small and possibly can be overcome by diffusion.

(ii) For small distances, on the other hand, the leading contribution is

$$
\begin{aligned}
& \frac{V\left(x_{0}\right)}{k_{\mathrm{B}} T}=\frac{Z \sigma \lambda_{\mathrm{B}}}{\kappa \mathrm{e}^{2}}\left(2 \pi-4 \cos \alpha \kappa x_{0} \ln \left|\kappa x_{0}\right|\right. \\
& \left.\quad+C(\cos \alpha) \kappa x_{0}+\mathrm{O}\left(\left(\kappa x_{0}\right)^{2}\right)\right),
\end{aligned}
$$

where $C(\cos \alpha)$ is a known function. For $x_{0}=0$ the value of $V\left(x_{0}\right)$ is positive and independent of $\alpha$, according to the superposition principle for two semi-infinite plates. Interestingly there is a logarithmic singularity for small $x_{0}$ which leads to an attractive force $-\mathrm{d} V\left(x_{0}\right) / \mathrm{d} x_{0}$ diverging as $-\ln \left(\kappa x_{0}\right)$ as $x_{0} \rightarrow 0$. The prefactor scales with $\cos \alpha$, i.e. it is maximal for small opening angles.

The full quantity $V\left(x_{0}\right)$ is plotted in units of its zerodistance limit $2 \pi k_{\mathrm{B}} T Z \sigma \lambda_{\mathrm{B}} / \mathrm{e}^{2} \kappa$ versus reduced distance $\kappa x_{0}$ in figure 9 for various opening angles $\alpha$. Clearly the attractive region for small $\kappa x_{0}$ and the repulsive region at large $\kappa x_{0}$ can be seen. The maximum in $V\left(x_{0}\right)$ occurs at $x_{0}^{c} \kappa=\mathrm{O}(1)$ the actual value depending on $\alpha$. Whether this attraction can be really felt by the macro-ion depends on its finite core size. If we assume a macro-ion hard core diameter of $d$, the condition $d<x_{0}^{c} \sin \alpha$ needs to be fulfilled in order to experience the effective attraction towards the wedge cusp.

The barrier to be overcome for escaping from the zerodistance minimum is of the order of roughly one in figure 9 , 


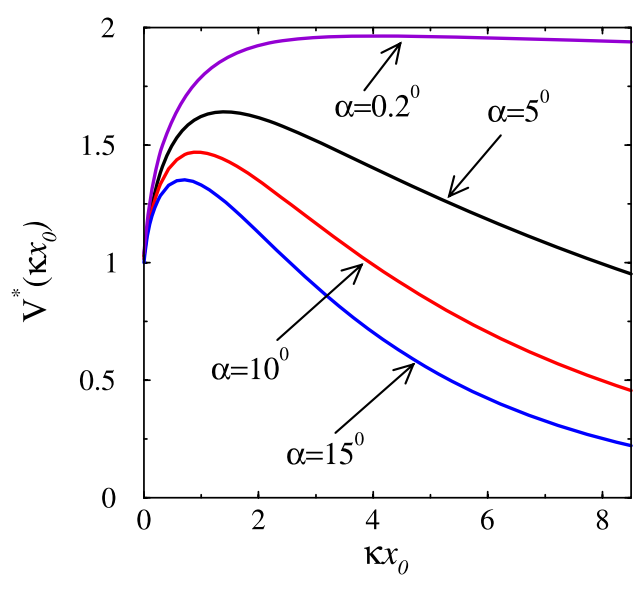

Figure 9. Reduced effective potential

$V^{*}\left(\kappa x_{0}\right)=V\left(x_{0}\right) \mathrm{e}^{2} \kappa / 2 \pi k_{\mathrm{B}} T Z \sigma \lambda_{\mathrm{B}}$ acting on a single macro-ion at a distance $x_{0}$ from the wedge cusp. The units of the potential are such that $V^{*}(0)=1 . V^{*}\left(\kappa x_{0}\right)$ is plotted versus reduced distance $\kappa x_{0}$ for three different opening angles $\alpha=0^{\circ}, 2^{\circ}, 5^{\circ}, 10^{\circ}, 15^{\circ}$.

(This figure is in colour only in the electronic version)

i.e. in units of $k_{\mathrm{B}} T$ it is of the order of $2 \pi Z \sigma \lambda_{\mathrm{B}} / \mathrm{e}^{2} \kappa$. The latter number can be of order one or much larger depending on the details of the charge densities etc.

One may also study an inverse wedge with opening angles $\alpha$ larger than $\pi / 2$. This corresponds formally to negative distances $x_{0}$ for a wedge of 'inflected' opening angle $\pi-\alpha$. In this situation the forces are always repulsive. The transition point $\alpha=\pi / 2$ is just a single planar plate where the exact solution of the Debye-Hückel theory [37] is

$$
\frac{V\left(x_{0}\right)}{k_{\mathrm{B}} T}=\frac{Z \sigma \lambda_{\mathrm{B}}}{\kappa e^{2}} 2 \pi \mathrm{e}^{-\kappa x_{0}} .
$$

In conclusion, within simple linear screening theory, there is an attractive region close to the wedge cusp of range $1 / \kappa$ but repulsion at larger distances. In fact, this can be understood qualitatively by a simple argument: the range of the screened Coulomb interaction is of the order of $1 / \kappa$. If one draws a sphere around the macro-ion (see figure 8) and compares all contributions from the wedge plates which are inside this sphere, there are two qualitatively different situations: if the macro-ion is close to the wedge cusp, more repulsion is coming from the right part, pushing the particles towards the wedge cusp. On the other hand, if the macro-ion is far away from the wedge, the repulsive plates are getting narrower towards the left which excludes the macro-ion from the wedge. This argument is not confined to linear wedges, it also applies to a to cone-like cusp ${ }^{4}$ or to a corner of a polyhedron.

\section{Primitive model computer simulation}

We have also approached the problem by computer simulation of the primitive model with explicit counter- and co-ions. Similar calculations have been performed to calculate the effective interaction between two spherical macro-ions [42, 43]

4 A cone-like geometry leads to a similar logarithmic singularity for $V(x)$ for small $x$ as in equation (3). and a single macro-ion near a single charged plate [44]. Here we model the charged wedge by two finite plates with onedimensional periodic boundary conditions in the cusp direction ( $z$ direction) of a periodicity length $L_{z}$. Perpendicular to this direction the wedge is closed by a neutral cylindrical hard wall which possesses a radial distance $L_{x}$ to the wedge cusp such that the cylinder radius of the hard wall is $L_{x}$. The total volume of the simulation box is $V_{\mathrm{s}}=\alpha L_{z} L_{x}{ }^{2}$. A similar set-up of a charged wedge was considered in [38] but there no macro-ion was included and the minimum image convention was used in the $y$ direction.

A macro-ion of charge $Z$ is placed at a distance $x_{0}$ from the cusp (see again figure 8). The macro-ion has a finite hard core diameter $d$ and releases $|Z / e|$ monovalent counterions of opposite charge $|e|$ and hard core diameter $d_{\mathrm{c}}$ in solution. These are considered together with the counterions stemming from the charged plates and the additional salt counter-and coions of bulk concentration $c_{\mathrm{s}}$. All micro-ions are monovalent and have the same hard core diameter $d_{\mathrm{c}}$. Interactions between the particles are taken within the primitive model to be a combination of Coulomb forces and excluded volume due to their hard cores. The effective force acting on the fixed macroion is calculated by an appropriate statistical average. By symmetry it is directed along the $x$ axis. The periodicity in the $z$ direction is taken into account by a one-dimensional Lekner summation [45].

The total electric field inside an empty wedge with charged surface in this geometry can be calculated analytically and reads as $\vec{E}(x, y, z)=\left(E_{x}(x, y), E_{y}(x, y), 0\right)$ with

$$
\begin{gathered}
E_{x}(x, y)=E_{1}\left(x_{1}, y_{1}\right) \cos \alpha-E_{2}\left(x_{1}, y_{1}\right) \sin \alpha \\
+E_{1}\left(x_{2}, y_{2}\right) \cos \alpha+E_{2}\left(x_{2}, y_{2}\right) \sin \alpha
\end{gathered}
$$

and

$$
\begin{gathered}
E_{y}(x, y)=E_{2}\left(x_{1}, y_{1}\right) \sin \alpha+E_{1}\left(x_{1}, y_{1}\right) \cos \alpha \\
+E_{2}\left(x_{2}, y_{2}\right) \sin \alpha-E_{1}\left(x_{2}, y_{2}\right) \cos \alpha
\end{gathered}
$$

with the notation $x_{1}=-x \sin \alpha+y \cos \alpha, x_{2}=x \sin \alpha+$ $y \cos \alpha, y_{1}=\sqrt{x^{2}+y^{2}-(y \cos \alpha-x \sin \alpha)^{2}}$, and $y_{2}=$ $\sqrt{x^{2}+y^{2}-(y \cos \alpha+x \sin \alpha)^{2}}$. Furthermore

$$
E_{1}(x, y)=\frac{\sigma}{2 \pi \epsilon_{0} \epsilon}\left(\arctan \left(\frac{L_{x} \cos \alpha-y}{x}\right)+\arctan \left(\frac{y}{x}\right)\right)
$$

and

$$
E_{2}(x, y)=\frac{\sigma}{4 \pi \epsilon_{0} \epsilon} \ln \left(\frac{x^{2}+y^{2}}{x^{2}+\left(L_{x} \cos \alpha-y\right)^{2}}\right) .
$$

In the absence of counterions, the lateral force in the wedge is always attractive towards the wedge cusp far away from the neutral curved wall and it diverges logarithmically with the size $L_{x} \cos \alpha$. Micro-ions will screen this field and could, in principle, even invert its sign.

In order to exclude spurious effects of the finite system sizes $L_{x}$ and $L_{z}$, we have calculated the force upon changing $L_{x}$ and $L_{z}$ both by a factor of 2 and found no change upon doubling. This shows that finite size effects are irrelevant in the distance region considered in this paper thanks to screening of the micro-ions, in particular salt ions. Hence 


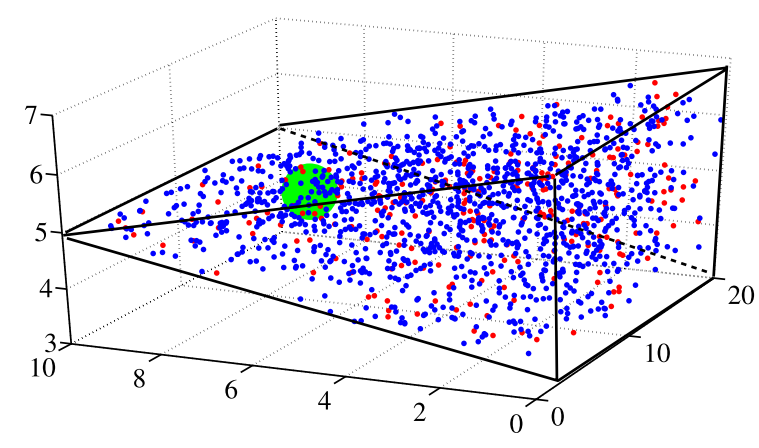

Figure 10. Simulation snapshot with $\alpha=10^{\circ}$ and $x_{0}=3 d$. The simulation parameters are: $d=10^{-7} \mathrm{~m}, d_{\mathrm{c}}=d / 150, Z=-60 e$, $\lambda_{\mathrm{B}}=7.8 \AA$, (i.e. $T=293 \mathrm{~K}$ and $\epsilon=80$ ), $c_{\mathrm{s}}=2.11 \times 10^{-7} \mathrm{~mol} \ell^{-1}$, $\sigma=-3.82 \times 10^{13} e \mathrm{~m}^{-2}$. All micro-ions are monovalent. The simulation box sizes were $L_{x}=160 d$ and $L_{z}=L_{x}$. The small light discs are co-ions, the darker discs are counterions. The macro-ion is almost completely squeezed between the wedge plates. Please note that only the inner part of the wedge is shown up to a distance of $10 \mathrm{~d}$ from the cusp. The lengths indicated are all in units of $d$.

we investigated single-macro-ion effects and any many-body effects stemming from the periodic images in the $z$ direction are strongly suppressed.

A simulation snapshot is presented in figure 10 where the macro-ion is almost squeezed completely between the wedge plates. The parameters used in the simulation are: $d=10^{-7} \mathrm{~m}$, $d_{\mathrm{c}}=d / 150, Z=-60 e, \lambda_{\mathrm{B}}=7.8 \AA$, (i.e. $T=293 \mathrm{~K}$ and $\epsilon=80), c_{\mathrm{s}}=2.11 \times 10^{-7} \mathrm{~mol} \ell^{-1}, \sigma=-3.82 \times 10^{13} e \mathrm{~m}^{-2}$. All micro-ions are monovalent. The simulation box sizes were $L_{x}=160 d$ and $L_{z}=L_{x}$. The parameters are typical for aqueous suspensions at very small salt concentration and weak plate charges.

Simulation results for the distance-resolved effective force $-\mathrm{d} V\left(x_{0}\right) / \mathrm{d} x_{0}$ acting on the macro-ion are compared to the predictions of linear screening theory in figure 11 . The value for $\kappa$ is taken from the Debye-Hückel expression where also the counterions from the walls and the macro-ion are accounted for via

$$
\kappa^{2}=4 \pi \lambda_{\mathrm{B}}\left(c_{\mathrm{c}}+2 c_{\mathrm{s}}\right)
$$

with the overall averaged counterion concentration $c_{\mathrm{c}}=$ $\left(2|\sigma / e| L_{z} L_{x} \cos \alpha+|Z / e|\right) / V_{\mathrm{s}}$ and the imposed salt concentration $c_{\mathrm{s}}$. All qualitative trends of the theory are confirmed, in particular the short-ranged attraction close to the cusp, while the actual numbers are in good agreement in the attractive region but differ more at larger distances. The latter fact can be understood by the fact that the theoretical screening parameters are taken to be too high since more micro-ions per volume are close to the apex of the wedge. Given the fact that the theory neglects the counterions from the macro-ions, the finite core of all particles, as well as nonlinear effects and any micro-ion correlations, the agreement is surprising.

\section{Discussion and conclusions}

In conclusion, we have addressed the question of macro-ion accumulation in a charge wedge by experiment, theory and computer simulation. The experiments reveal that a significant

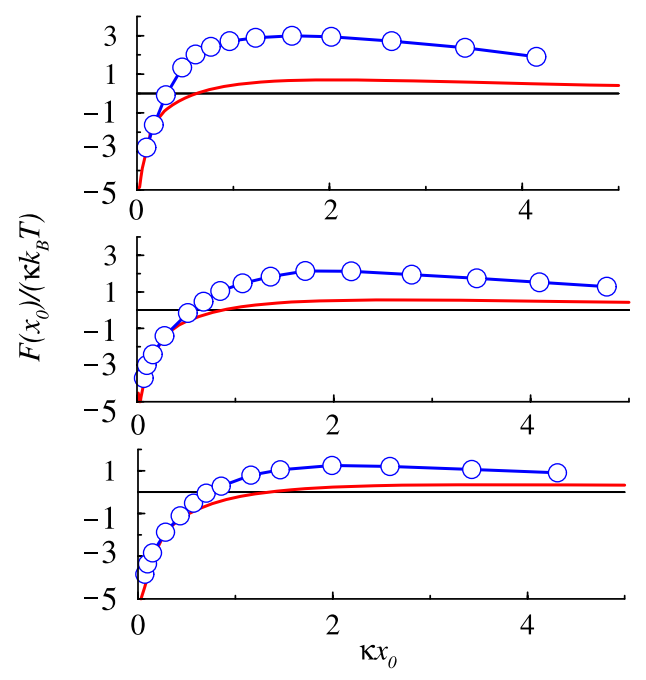

Figure 11. Effective force $F=-\mathrm{d} V\left(x_{0}\right) / \mathrm{d} x_{0}$ acting on a single macro-ion at a distance $x_{0}$ from the wedge cusp. The force is given in units of $\kappa k_{\mathrm{B}} T$ and shown versus reduced distance $\kappa x_{0}$ for the three different opening angles (from bottom to top): $\alpha=5^{\circ}, 10^{\circ}, 15^{\circ}$. Symbols (circles) are simulation data, the solid line is the linear screening theory. The simulation parameters are as in figure 10 . The averaged Debye screening length is $1 / \kappa=7 d$ which was taken as an input in the linear screening theory and is used as a length unit in the plots.

fraction of colloidal particles accumulates in the inner wedge where they form a dense layer. It was carefully checked that this is not caused by evaporation currents nor by salt gradients. The accumulation effect was found to be robust with respect to different wedges, opening angles, salt concentrations and occurs even in the presence of additional convection fields. The linear screening theory performed for a single particle predicts an attractive 'trapping' force towards the cusp of the wedge. The force diverges logarithmically in particle distance to the wedge. The range of the attraction is of the order of the Debye-Hückel screening length but increases strongly for very small opening angles of the wedge. The simple linear screening theory was tested against micro-ion-resolved computer simulations and qualitative and semi-quantitative agreement was found.

The most notable difference between our theoretical calculations and the experiments is that the theory is based on a single particle while in most of the experiments many particles are present. This is the reason why a direct comparison is less obvious. Two more direct ways are as follows; (1) perform additional experiments on individual particles close to the cusp using laser tweezers, which would allow a direct measurement of lateral forces, (2) perform the theory and the simulations at finite (higher) concentrations such that many-body effects are included. While we have started to work along both of these lines, we can at the moment bring together the experiments and the theory only in a qualitative way.

For many particles, the leading effect is still expected to result in an attractive trapping force close to the wedge with a longer-ranged small potential barrier for larger distances. The particles will surmount this small potential energy barrier by the large osmotic pressure of the colloidal reservoir. The 
trapping force then helps to stabilize and finalize the effect insofar as a significant concentration of particles is found close to the wedge edges. The trapping force may also be thought of as acting and being amplified in an effective way; once a fraction of particles has already accumulated in the wedge the particles following may see an effective wedge formed by those particles such that they again effectively feel the trapping force which then consistently leads to an accumulation of colloids into the wedge. This picture, of course, is merely qualitative and needs more quantitative support, in particular from the theoretical side.

More concretely, the theory explains at least three facts clearly: first, in an inverted cell where gravity pushes out colloidal particles from the wedge cusp, it took a long time until all particles did disappear from the wedge cusp. The area density close to the wedge cusp was larger than further away where a depletion zone was visible. These experiments work in the low density regime where the theory is applicable. The experiments therefore clearly reveal the existence of an attractive trapping force as predicted by the theory. This is the most direct comparison between experiment and theory. Second, after strong convection there is still a particle layer trapped close to the wedge edges. In fact the convection flow tries to remove particles from the inner wedge but the trapping force keeps them there. Hence, the linear screening theory explains the observed large depletion zone of colloidal particles between the dense inner region and the concentration outer region. The depletion zone should coincide more or less with the region around the potential barrier. The fact that colloidal particles have to surmount a barrier might explain why it takes a long time to achieve the final depletion region experimentally. Third, the linear screening theory also explains that the accumulation effects are there but less pronounced for increased salt concentration (larger $\kappa$ ) since the range and strength of the attraction decrease with growing $\kappa$.

We emphasize that - in order to explain particle accumulation in the wedge - one does not need to invoke a like-charge attraction picture [18]. In fact, the linear screening theory is based on like-charge repulsion. In a first instance it is tempting to attribute the accumulation effect to a long-ranged likecharge attraction but we think that it is rather a combined effect of osmotic pressure plus the small potential barrier and the trapping force.

Our findings have important implications for particle transport through narrow channels. At edges of the channels, particles may get trapped due to the attractive force and this may block further particle transport. Furthermore, in cuspy charged surfaces where the surface roughness is on a micronscale, particles may delve into deep surface holes and stay there for a long time. The attractive force is even more dominant in low-dielectric constant solvents $(\epsilon=5-6)$ as found in organic solvents [46-48] or in complex plasmas [49]. There the range of the attraction can extend over several interparticle layer spacings such that the trapping force will lead to more pronounced effects.

As already mentioned, remaining issues are the development of a theory and a simulation at finite colloidal densities and of parameters relevant for the experimental samples. As discussed above, we have focused here on a first qualitative understanding of the mechanisms rather than on a quantitative comparison of numbers and we leave the latter for the future. In fact, it would be interesting to extend the theory to many macro-ions. This should first be done on the linear screening level where the macro-ions interact via a pairwise Yukawa potential $[43,50]$. On a higher level of complexity, one may perform extended computer simulations of the primitive model for more macro-ions. This would allow for a systematic understanding of many-body effects and for a more quantitative comparison between experiment and theory. We would expect that layering transitions would then play an important role, similar to those found for neutral particles in neutral wedges [40, 41]. Then one could, in principle, think about using charged colloids in charged wedges as a model system for wetting transitions such as the wedge filling transition [51-53].

\section{Acknowledgments}

We thank R Messina and E C Oğuz for helpful discussions. This work is supported by the DFG within SFB TR6 (project section D1).

\section{References}

[1] Dietrich S and Haase A 1995 Phys. Rep. 260

[2] Drake J M and Klafter J 1990 Phys. Today 4346

[3] Schmidt M and Löwen H 1997 Phys. Rev. E 557228

[4] Murray C A and van Winkle D H 1987 Phys. Rev. Lett. 581200

[5] Löwen H 2001 J. Phys.: Condens. Matter 13415

[6] Helseth L E, Wen H Z and Fischer T M 2006 J. Appl. Phys. 99024909

[7] Unni H N, Keh H J and Yang C 2007 Electrophoresis 28658

[8] Liu D, Maxey M and Karniadakis G E 2004 J. Micromech. Microeng. 14567

[9] Murray C A and Grier D G 1996 Annu. Rev. Phys. Chem. 47421

[10] Brunner M, Bechinger C, Strepp W, Lobaskin V and von Grünberg H H 2002 Europhys. Lett. 58926

[11] Weiss J A, Larsen A E and Grier D G 1998 J. Chem. Phys. 1098659

[12] Crocker J C and Grier D G 1994 Phys. Rev. Lett. 73352

[13] Chakrabarti J and Löwen H 1998 Phys. Rev. E 583400

[14] Allahyarov E, D'Amico I and Löwen H 1999 Phys. Rev. E 603199

[15] Messina R and Löwen H 2003 Phys. Rev. Lett. 91146101

[16] Barreira Fontecha A, Schöpe H J, König H, Palberg T, Messina R and Löwen H 2005 J. Phys.: Condens. Matter 172779

[17] Krishnan M, Mönch I and Schwille P 2007 Nano Lett. 71270

[18] Baumgartl J, Arauz-Lara J L and Bechinger C 2006 Soft Matter 8631

[19] Neu J C 1999 Phys. Rev. Lett. 821072

[20] Sader J E and Chan D Y C 2000 Langmuir 16324

[21] Löwen H, Messina R, Hoffmann N, Likos C N, Eisenmann C, Keim P, Gasser U, Maret G, Goldberg R and Palberg T 2005 J. Phys.: Condens. Matter 17 S3379

[22] Barreira Fontecha A, Schöpe H J and Palberg T 2007 Phys. Rev. E 76 050402(R)

[23] Ramiro-Manzano F, Bonet E, Rodriguez I and Meseguer F 2007 Phys. Rev. E 76050401

[24] Pieranski P, Strzlecki L and Pansu B 1983 Phys. Rev. Lett. 50 900 
[25] Lyklema H 1993-2000 Fundamentals of Interface and Colloid Science vol 1-3 (London: Academic)

[26] Yamanaka J, Murai N, Iwayama Y, Yonese M, Ito K and Sawada T 2004 J. Am. Chem. Soc. 1267156

[27] Iacopini S, Rusconi R and Piazza R 2006 Eur. Phys. J. E 1959

[28] Neser S, Bechinger C, Leiderer P and Palberg T 1997 Phys. Rev. Lett. 792348

[29] Wette P, Schöpe H J, Biehl R and Palberg T 2001 J. Chem. Phys. 1147556

[30] Schöpe H J, Barreira Fontecha A, Knig H, Marques Hueso J and Biehl R 2006 Langmuir 221828

[31] Schöpe H J 2003 J. Phys.: Condens. Matter 15 L533

[32] Palberg T and Würth M 1994 Phys. Rev. Lett. 72786

[33] Dhukin S S and Derjaguin B V 1974 Surface and Colloid Science vol 7, ed E Matijevic (New York: Wiley)

[34] Deegan R D, Bakajin O, Dupont T F, Huber G, Nagel S R and Witten T A 1997 Nature 389827

[35] Okubo T, Nakagawa N and Tsuchida A 2007 Colloid Polym. Sci. 2851247

[36] Löwen H, Hansen J P and Madden P A 1993 J. Chem. Phys. 983275

[37] Hansen J P and Löwen H 2000 Annu. Rev. Phys. Chem. 51209

[38] Koenders M A C and Reymann S 2000 J. Colloid Interface Sci. 225299

[39] Kang K H, Kang I S and Lee C M 2003 Langmuir 199334
[40] Tata B V R, Boda D, Henderson D, Nikolov A and Wasan D T 2000 Phys. Rev. E 623875

[41] Trokhynichuk A, Henderson D, Nikolov A and Wasan D T 2005 Langmuir 2110240

[42] Allahyarov E, D'Amico I and Löwen H 1998 Phys. Rev. Lett. 811334

[43] Allahyarov E, Löwen H and Trigger S 1998 Phys. Rev. E 575818

[44] Allahyarov E, D’Amico I and Löwen H 1999 Phys. Rev. E 603199

[45] Gronbech-Jensen N, Hummer G and Beardmore K M 1997 Mol. Phys. 92941

[46] Royall C P, Leunissen M E and van Blaaderen A 2003 J. Phys.: Condens. Matter $15 \mathrm{~S} 3581$

[47] Campbell A I, Anderson V J, van Duijneveldt J S and Bartlett P 2005 Phys. Rev. Lett. 94208301

[48] Lu P J, Conrad J C, Wyss H M, Schofield A B and Weitz D A 2006 Phys. Rev. Lett. 96028306

[49] Morfill G E, Ivlev A V, Rubin-Zuzic M, Knapek C A, Pompl R, Antonova T and Thomas H M 2007 Appl. Phys. B 89527

[50] Bitzer F, Palberg T, Löwen H, Simon R and Leiderer P 1994 Phys. Rev. E $\mathbf{5 0} 2821$

[51] Rejmer K, Dietrich S and Napiorkowski M 1999 Phys. Rev. E 604027

[52] Müller M and Binder K 2005 J. Phys.: Condens. Matter 17333

[53] Rascon C and Parry A O 2005 Phys. Rev. Lett. 94096103 